\title{
Knockdown of microRNA-143-5p by STTM technology affects eumelanin and pheomelanin production in melanocytes
}

\author{
SHUHUI QI, BO LIU, JUNZHEN ZHANG, XUEXIAN LIU, CHANGSHENG DONG and RUIWEN FAN \\ College of Animal Science and Veterinary Medicine, Shanxi Agricultural University, Taigu, Shanxi 030801, P.R. China
}

Received January 15, 2019; Accepted June 12, 2019

DOI: $10.3892 / \mathrm{mmr} .2019 .10492$

\begin{abstract}
MicroRNAs (miRNAs) serve various roles in the regulation of melanogenesis in mammalian melanocytes that contribute to the development of hair color. The manipulation of the melanocyte action is a new target for genetic improvement. Short tandem target mimic (STTM) is a potent approach for silencing miRNAs in plants and animals. To investigate the function of miR-143-5p in melanogenesis, STTM was used to block the expression of miR-143-5p (STTM-miR-143-5p). The molecular analysis and luciferase reporter assay identified myosin Va gene (MYO5A) as one of the miR-143-5p targets. STTM-miR-143-5p overexpression resulted in an increased expression of downstream melanogenesis genes including microphthalmia-associated transcription factor $(M I T F)$, tyrosinase family members [tyrosinase $(T Y R)$ and tyrosinase-related protein 1(TYRP1)], melanophilin $(M L P H)$, and Rab27a, thereby contributing to melanocyte pigmentation by promoting total alkali-soluble melanogenesis (ASM) and eumelanin (EM) contents; conversely, STTM-miR-143-5p overexpression resulted in decreased expression of the tyrosinase-related protein 2 (TYRP2)/dopachrome tautomerase (DCT), which is responsible for decreased pheomelanin (PM) content in mouse melanocytes. The results indicated that melanin production in melanocytes could be increased by manipulating miR-143-5p expression using STTM which resulted in ASM and EM production.
\end{abstract}

\section{Introduction}

The phenotype of hair color depends on the levels and ratio of two types of melanin, namely black-brown eumelanin (EM) and yellow-red-brown pheomelanin (PM), which are produced by

Correspondence to: Professor Changsheng Dong or Professor Ruiwen Fan, College of Animal Science and Veterinary Medicine, Shanxi Agricultural University, Taigu, 1 Mingxian South Road, Taigu, Shanxi 030801, P.R. China

E-mail: csdong18@163.com

E-mail: ruiwenfan@163.com

Key words: short tandem target mimic, miR-143-5p, myosin Va, eumelanin, pheomelanin, melanocyte resident skin melanocytes (1). The hair color formation includes melanin production in melanocytes and melanin transport to keratinocytes (2). Several genes and miRNAs are involved in the pathways of melanin production. microphthalmia-associated transcription factor (MITF) is an important transcription factor that activates the key limiting enzymes tyrosinase (TYR), tyrosinase-related protein 1 (TYRP1), and tyrosinase-related protein 2(TYRP2)/DCT for the synthesis and storage of melanin in melanosomes $(3,4)$. During melanin transportation, the tripartite complex Rab27a/melanophilin (MLPH)/myosin Va (MYO5A) plays a crucial role (5) and acts as a linker in the entire protein complex $(6,7)$. The complex is required for connecting the melanosome to the actin cytoskeleton in order to facilitate the normal accumulation of the organelle in the dendritic tips (8). MYO5A is an actin-based molecular motor typically involved in the transport of organelles and vesicles, such as the melanosomes $(9,10)$. MYO5A can bind to more than one type of cellular structure and its selectivity is determined by alternate spliced sequences in melanocytes (11).

Short tandem target mimics (STTMs) are based on target mimicry (TM) and aim to block the function of small RNA molecules in animals and plants (12). The expression of the microRNA-143 (miR-143) cluster is required in various types of cells. As predicted by the TargetScan analysis, miR-143-5p targets MYO5A. In the present study, the STTM method was used, which is considered a powerful technology to complement existing small RNA sequestration (12). An STTM-miR-143-5p plasmid was constructed to inhibit the function of miR-143-5p in the regulation of EM and PM production in mouse melanocytes.

\section{Materials and methods}

Construction of plasmids. An oligonucleotide containing the STTM-miR-143-5p sequence was chemically synthesized according to a previously described method (12). The oligonucleotide was inserted into the dual-luciferase vector, pmirGLO (Promega Corporation) in order to construct the expression plasmid pmirGLO-STTM-miR-143-5p, in which the CMV promoter was used to induce GFP and STTM-miR-143-5p expression. The null pmirGLO vector was used as the corresponding negative control (NC). The luciferase reporter expression plasmid was constructed by cloning the 3'-UTR sequence of the mouse MYO5A into the dual luciferase pmirGLO vector (Promega Corporation). The partial 
sequence of the mouse MYO5A containing the miR-143-5p binding sites was obtained by PCR with mouse melanocyte cDNA as the template and the primers that contained the XhoI and SacI sites (Table I). The PCR product was subsequently digested with XhoI and $S a c I$ and cloned into the $S a c I$ and $X h o I$ restriction sites of the vector in order to produce the pmirGLO-MYO5A-wt plasmid. In addition, the MYO5A 3'-UTR with the miR-143-5p binding sites was mutated using a site-directed gene mutagenesis kit (Beyotime Institute of Technology) to generate the pmiGLO-MYO5A-mut plasmid. All constructs were verified by sequencing (Shenzhen Huada Gene Co., Ltd.).

Melanocyte transfection. The mouse melanocytes used in the present study were established and maintained in our laboratory (13). The melanocytes were transfected with the pmirGLO-STTM-miR-143-5p plasmid and/or the pmirGLO null plasmid (NC) using the Lipofectamine 2000 assay (Invitrogen; Thermo Fisher Scientific, Inc.) following the manufacturer's instructions. The melanocytes were collected two days after, following transfection for cell lysis and total RNA preparation.

Dual luciferase reporter assay for miRNA target validation. 293T cells were cultured in DMEM (Gibco; Thermo Fisher Scientific, Inc.) supplemented with $10 \%$ fetal bovine serum (FBS) and were transfected with $580 \mathrm{ng}$ of pmirGLO-MYO5A-wt or pmirGLO-MYO5A-mut plasmids in the presence of $20 \mathrm{ng}$ of pmirGLO-STTM-miR-143-5p or pmirGLO-NC plasmids using Lipofectamine 2000. These preparations were used for the luciferase assays two days after co-transfection. Luciferase activity was subsequently measured using the Dual-Luciferase Reporter Assay kit (Promega Corporation). The firefly luciferase activity of each test sample was normalized to that of Renilla. The data were performed in triplicate and expressed as the mean relative luciferase activity (mean $\pm \mathrm{SD}$ ).

Reverse transcription-quantitative polymerase chain reaction (RT-qPCR) analysis of miRNA and mRNA expression levels. Total RNA from melanocytes was extracted using TRIzol reagent (Invitrogen; Thermo Fisher Scientific, Inc.) following the manufacturer's instructions and treated with DNase I (Sigma-Aldrich; Merck KGaA). For quantitative mRNA expression analysis, $1 \mu \mathrm{g}$ of total RNA was reverse-transcribed to cDNA using a cDNA synthesis kit (Takara Bio, Inc.) as described in the manufacturer's instructions. The samples were amplified by quantitative real time PCR using SYBR-Green PCR master mix (Takara Bio, Inc.). For quantitative miRNA expression analysis, cDNA was produced using a specific stem-loop RT primer pair, a common reverse primer (Table I) pair and a cDNA synthesis kit (Takara Bio, Inc.) according to a previously described method (14). SYBR-Green PCR master mix (Takara Bio, Inc.) was used for RT-qPCR reactions performed on a 7500 Fast Real-Time PCR ${ }^{\mathrm{TM}}$ system (Applied Biosystems Life Technologies; Thermo Fisher Scientific, Inc.). The relative expression levels of mRNA and miRNA were quantified using the quantification cycle $(\mathrm{Cq})$ method (15) and the normalization was performed with regard to the amounts of $\beta$-actin and $U 6$ mRNA, respectively.
Western blot analysis. The cell lysates from melanocytes were obtained using RIPA cell lysis reagent (Beyotime Institute of Biotechnology) and western blotting was conducted as previously described (16). The following primary antibodies were used: Anti-MYO5A (rabbit resource) at a 1:1,000 dilution (cat.no. 3402; RRID:AB_2148475; Cell Signaling Technology, Inc.), anti-MLPH (mouse resource) at a 1:1,000 dilution (cat. no. 66092-1-Ig; RRID:AB_11232039; ProteinTech Group), anti-RAB27A (mouse resource) at a 1:1,000 dilution (cat. no. ab55667; RRID:AB_945112), anti-MiTF (rabbit resource) at a 1:1,000 dilution (cat. no. ab20663; RRID:AB_470315), anti-TYR (rabbit resource) at a 1:1,000 dilution (cat. no. ab61294), anti-TYRP1 (rabbit resource) at a 1:1,000 dilution (cat. no. ab83774; RRID:AB_2211142), and anti-TYRP2 (rabbit resource) at a 1:1,000 dilution (cat. no. ab74073; AB_1524517; all from Abcam), and anti-GAPDH (rabbit resource) at a 1:10,000 dilution (cat. no. AP0063, Bioworld Technology, Inc.). Anti-rabbit IgG secondary antibodies (cat. no. CW0103) and anti-mouse IgG secondary antibodies (cat. no. CW0102) were commercially purchased. The immunoblots were exposed to develop the chemicals and subsequently scanned using a ChemiDOC ${ }^{\mathrm{TM}}$ XRS + imager (Bio-Rad Laboratories, Inc.). Protein expression was quantified from the band intensity which was assessed by densitometry using the Image-Pro Plus software (Olympus Corporation).

Spectrophotometric assay of melanin content. Following transfection, the harvested melanocytes were rinsed with phosphate-buffered saline (PBS). The spectrophotometric assay of the alkali-soluble melanin required melanocytes that were lysed with $1 \mathrm{ml}$ of $1 \mathrm{M} \mathrm{NaOH}$ at $37^{\circ} \mathrm{C}$. The cells were incubated for $1 \mathrm{~h}$ and the absorbance was measured at $475 \mathrm{~nm}$ spectrophotometrically. The determination of EM was achieved by melanocyte hydrolysis at $80^{\circ} \mathrm{C}$ in $30 \%$ hydroiodic acid and 30\% hypophosphoric acid. Following cooling and the addition of $50 \%$ ethanol, the samples were centrifuged at 2,234 x g for $10 \mathrm{~min}$ in order to collect the insoluble eumelanic pigments that were subsequently solubilized at $80^{\circ} \mathrm{C}$ in hydrogen peroxide and sodium hydroxide. Following centrifugation at $10,700 \mathrm{x} \mathrm{g}$ for $1 \mathrm{~min}$ in a Sorvall Ultracentrifuge, the supernatant was obtained and its absorbance was measured at $350 \mathrm{~nm}$. The concentration of PM was determined in transfected melanocytes that were solubilized in phosphate buffer ( $\mathrm{pH} 10.5)$ and centrifuged at 10,700 x g for $10 \mathrm{~min}$. The supernatant was obtained and the absorbance was recorded at $400 \mathrm{~nm}$. The melanin contents were normalized to the total numbers of cells. All experiments were performed in triplicate.

Fontana-Masson staining. Following melanocytes transfection with STTM-miR-143-5p or pmirGLO null vector, the plasmids were washed thoroughly with PBS ( $\mathrm{pH} 7.4)$ and the cells were fixed in $4 \%$ formaldehyde for $20 \mathrm{~min}$. The cells were subsequently stained using Ammoniacal Silver solution from the Fontana-Masson stain kit (Abcam) at $55^{\circ} \mathrm{C}$ in the dark for $1 \mathrm{~h}$, and washed with distilled water thoroughly thereafter. The cells were further stained using $0.2 \%$ gold chloride solution and 5\% sodium thiosulfate solution for $2 \mathrm{~min}$ and counter-stained with Nuclear Fast Red solution for $5 \mathrm{~min}$. The 
Table I. Primers used in the present study.

\begin{tabular}{|c|c|c|}
\hline Primer name & Primer sequence $5^{\prime}-3^{\prime}$ & Application \\
\hline MYO5A-F & AAAATGCTGCGGTTAGGA & RT-qPCR \\
\hline MYO5A-R & GCTTGGGAGGTATTGTGC & RT-qPCR \\
\hline MYO5A-wt-F & CGAGCTCAAAATGCTGCGGTTAGG & Luciferase reporter-wt \\
\hline MYO5A-wt-R & CCGCTCGAGCCAGTTAAAGAGTTTTGCATAG & Luciferase reporter-wt \\
\hline MYO5A-mut-F & TACCTGCAGATGCACCTCTGCAAGTAGCAGACACTGG & Luciferase reporter-mut \\
\hline MYO5A-mut-R & CCAGTGTCTGCTACTTGCAGAGGTGCATCTGCAGGTA & Luciferase reporter-mut \\
\hline miR-143-5p-RT & CTCAACTGGTGTCGTGGAGTCGGCAATTCAGTTGAGACCAGAGA & RT-qPCR \\
\hline miR-143-5p-F & ACACTCCAGCTGGGGGTGCAGTGCTGCATC & RT-qPCR \\
\hline Common-R & CGAGCAGTGCAGGGTCCGAGGT & RT-qPCR \\
\hline U6-RT & GTCGTATCCAGTGCAGGGTCCGAGGTATTCGCACTGGATACGACTCATCT & RT-qPCR \\
\hline U6-F & CTCGCTTCGGCAGCACA & RT-qPCR \\
\hline MLPH-F & GGTTTCCTGCTTCTGTTCG & RT-qPCR \\
\hline MLPH-R & CGTGGCTTATGTTTGTCCC & RT-qPCR \\
\hline Rab27a-F & TATGGGTTTCCTGCTTCT & RT-qPCR \\
\hline Rab27a-R & GCCTCCTCСТCTTTCACT & RT-qPCR \\
\hline MITF-F & TGAGGAGCAGAGCAGGGCAGAGAGT & RT-qPCR \\
\hline MITF-R & TGGGAAGGTTGGCTGGACAGGAGTT & RT-qPCR \\
\hline TYR-F & TGAAAATCCTAACTTACTCAGCCCA & RT-qPCR \\
\hline TYR-R & TCAAACTCAGACAAAATTCCACATC & RT-qPCR \\
\hline TYRP1-F & CCATTGCTGTAGTGGCTGCGTTGTT & RT-qPCR \\
\hline TYRP1-R & GGAGAGGCTGGTTGGCTTCATTCTT & RT-qPCR \\
\hline TYRP2-F & TTGCTCTTGGGGTTGCTGGCTTTTC & RT-qPCR \\
\hline TYRP2-R & TCCTCCGTGTATCTCTTGCTGCTGA & RT-qPCR \\
\hline$\beta$-actin-F & TTGCTGACAGGATGCAGAAG & RT-qPCR \\
\hline$\beta$-actin-R & ACATCTGCTGGAAGGTGGAC & RT-qPCR \\
\hline
\end{tabular}

MYO5A, myosin Va; F, forward; R, reverse; wt, wild-type; mut, mutant; MLPH, melanophilin; MITF, microphthalmia-associated transcription factor; TYR, tyrosinase; TYRP1, tyrosinase-related protein 1; TYRP2, tyrosinase-related protein 2; RT-qPCR, reverse transcription-quantitative polymerase chain reaction.

samples were washed with distilled water following every staining process. The melanin content was visualized under a light microscope.

Statistical analysis. The difference in melanin production, the levels of miRNA, mRNA and protein, and the relative luciferase activities were analyzed using ANOVA and the protected Fisher's least significant difference tests. The analysis was conducted using SPSS 11.5 software (SPSS, Inc.) and the differences were determined using a P-value threshold of $0.05(\mathrm{P}<0.05)$

\section{Results}

STTM-miR-143 reduces the levels of $m i R-143$ and its binding to MYO5A in melanocytes. To evaluate whether STTM-miR-143-5p affected miR-143 activity, the expression levels of miR-143 and its predicted target gene MYO5A were investigated in melanocytes transfected with pmirGLO-miR-143 by RT-qPCR. The results indicated that miR-143 expression was significantly decreased and that MYO5A expression was significantly increased following STTM-miR-143-5p treatment (Fig. 1).
To determine whether binding to conserved target sites could cause a reduction in the expression levels of MYO5A (Fig. 2A and B), the plasmids containing the 3'-UTR of MYO5A were co-transfected into melanocytes with pmirGLO-STTM-miR-143 or the corresponding pmirGLO null vector. Co-transfection of the pmirGLO-STTM-miR-143 and the pmirGLO-MYO5A-wt plasmids or of the empty vector and the pmirGLO-MYO5A-wt plasmids into the cells indicated that pmirGLO-STTM-miR-143 significantly increased the luciferase activity of wild-type of MYO5A. Furthermore, luciferase reporter assays indicated that point mutations in the sequences of pmirGLO-MYO5A-mut abolished the effects of endogenous miR-143 by reducing complementarity between miR-143 and the target sites of MYO5A (Fig. 2C and D). These data indicated that miR-143 could bind to the 3'-UTR of MYO5A and significantly inhibit its expression.

Effect of STTM-miR-143 overexpression on the melanogenic gene expression. The melanocytes that were transfected with the pmirGLO-STTM-miR-143 and negative control plasmids were harvested in order to examine the levels of the melanogenic genes, namely $T Y R, T Y R P 1$ and $T Y R P 2$. In 
A

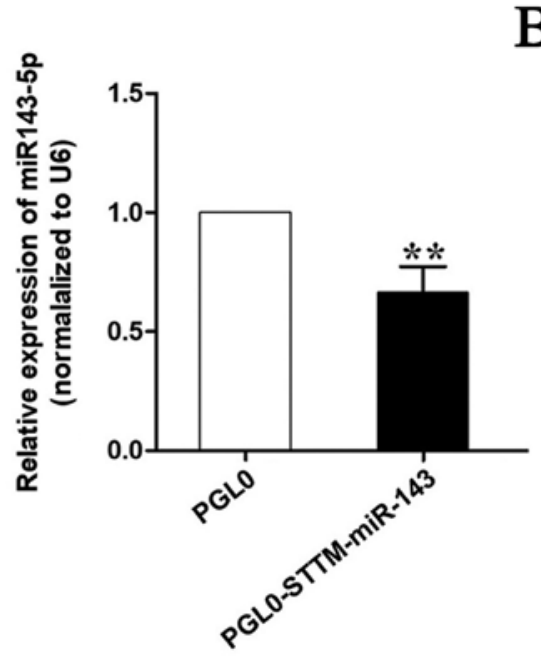

C

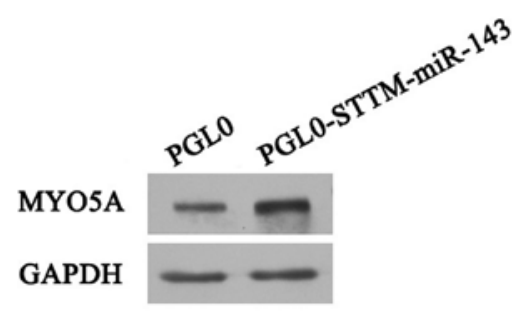

B

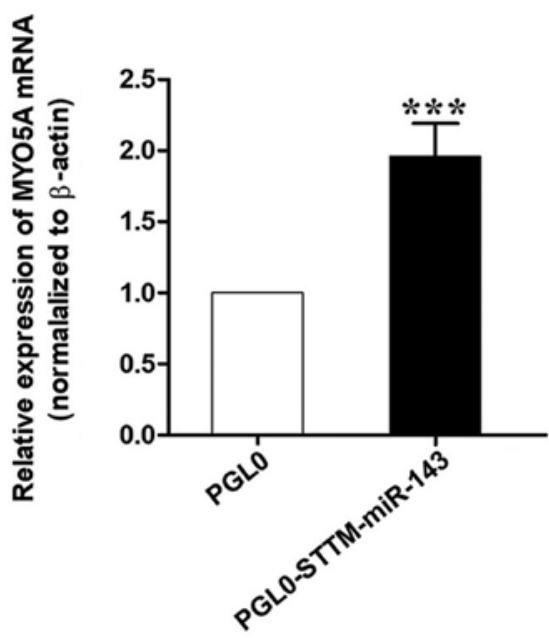

D

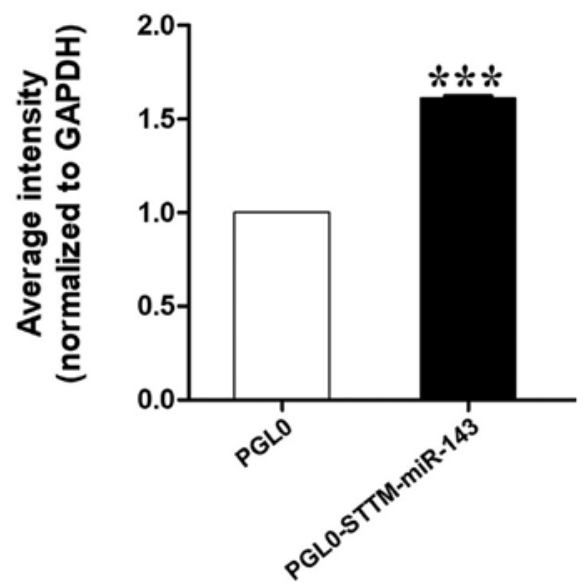

Figure 1. Effects of STTM-miR-143-5p on the expression levels of miR-143-5p and MYO5A. (A) The expression levels of miR-143-5p in mouse melanocytes transfected with the STTM-miR-143-5p expression plasmids. (B) The mRNA expression levels of MYO5A in melanocytes transfected with the STTM-miR-143-5p plasmids. (C and D) Analysis of MYO5A protein expression in mouse melanocytes transfected with the miR-143-5p expression plasmids by western blotting. The data are expressed as the mean $\pm \mathrm{SD}(\mathrm{n}=3)$. ${ }^{* *} \mathrm{P}<0.01 ;{ }^{* * *} \mathrm{P}<0.001$ vs. PGL0. STTM, short tandem target mimic; MYO5A, myosin Va.

addition, the levels of the transcription factor MITF, and of the melanin transport genes $M L P H$, and Rab27a were examined. RT-qPCR and western blotting analyses demonstrated that overexpression of pmirGLO-STTM-miR-143 in melanocytes significantly increased the mRNA and protein levels of MLPH, Rab27a, MITF, TYRP1 and TYR. Conversely, overexpression of pmirGLO-STTM-miR-143 in melanocytes significantly decreased the mRNA and protein levels of Tyrp2 (Fig. 3).

Effects of STTM-miR-143 overexpression on melanin production. To determine whether pmirGLO-STTM-miR-143 overexpression could affect melanin production, melanocytes transfected with pmirGLO-STTM-miR-143 plasmid were harvested and total alkali-soluble melanogenesis (ASM), pheomelanogenesis (PM), and eumelanogenesis (EM) were measured.The results indicated that pmirGLO-STTM-miR-143 overexpression in mouse melanocytes increased ASM and PM by $30 \%(\mathrm{P}<0.01$; Fig. 4A and $\mathrm{C})$, while it decreased EM by approximately $20 \%(\mathrm{P}<0.01$; Fig. 4B). Fontana-Masson staining confirmed the localization of melanin in the melanocytes. Overexpression of the pmirGLO-STTM-miR-143-5p plasmids in melanocytes significantly increased the melanin content and distribution compared with those noted in the negative control (Fig. 4D).

\section{Discussion}

Genomic analysis has been previously used to investigate several aspects of pigment cell biology, including melanocyte- and melanosome-associated functions, pigment disorders, normal pigmentation variation and even melanoma (17). miRNAs are regulators of cellular events, such as differentiation, proliferation, and reprogramming (18), and have often been implicated in the regulation of melanogenesis for the determination of hair color (19). Our previous study identified miR-143 as a negative regulator of proliferation, migration and melanogenesis in mouse melanocytes by direct targeting of the TGF- $\beta$-activated kinase 1 (TAK1) (20). However, the effects of miR-143 knockdown on melanogenesis have not been previously identified. STTM is a powerful technology used to block miRNA function in animals (12). Our previous studies utilized STTM technology in order to inhibit miRNA-508-3p and demonstrated its efficacy in upregulating melanogenesis (21). In the present study, the 


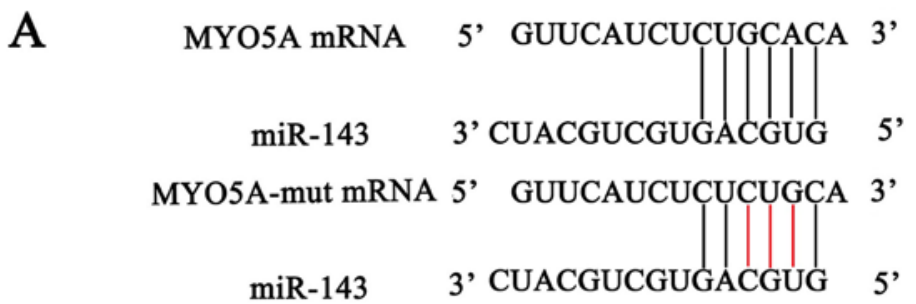

B Rabbit UCAUCGAAAAUAGUGAUAUAGUUAACCUUUGAAAUGUUUUUAGGUG
Cow ACAUGGAAAAACAUGAUCUAGUUAAUCUUGGAAAGUUCUUAAGUG
Mouse AGAAUUUUCUUGACCUACACAGUUCAUCUCUGCACA GUGGUUAGUUG
Human AUCUGCAUAGAAAAUUUUGAUACAAUUUUUGAAAGUCUUAGGUG
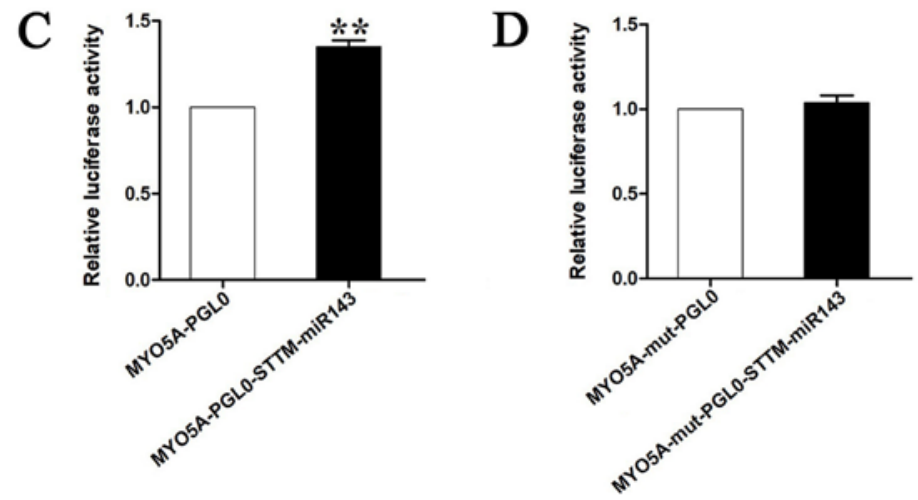

Figure 2. The 3'-UTR of MYO5A transcript is a target for miR-143-5p. (A) The miR-143-5p binding sites in the 3'-UTR of MYO5A were predicted. (B) The alignment of the MYO5A sequences around the miR-143-5p binding sites (seed region is indicated by the box). (C and D) Luciferase reporter assays in 293T cells co-transfected with the wild-type 3'-UTR of MYO5A (pmirGLO-MYO5A-wt/mut) and the miR-143-5p or the NC plasmids. The data from dual luciferase assays are expressed as the mean relative luciferase activities $\pm \mathrm{SD}(\mathrm{n}=3) .{ }^{* *} \mathrm{P}<0.01$ vs. MYO5A-PGL0. MYO5A, myosin Va.

same methodology was used to inhibit miR-143 expression in melanocytes.

Melanocytes located in the skin are derived directly from the neural crest cells and the embryonic cells that are present in the skin by the dorsolateral migration pathway (22). They are under the modulation of intrinsic factors and are controlled by extracellular signals (22). miR-143 plays a key role in embryonic stem cell (ESC) pluripotency (23), by targeting the sex-determining region Y-box 2 (SOX2), the Krüppel-like factor 4 (KLF4), and the octamer binding transcription factor 4 (OCT4) (20). These functions indicate that miR-143 promotes ESC differentiation into different cell lineages, such as neural crest cells (20). In mammalian skin, melanocytes produce melanin and form mature melanosomes that complete their pigmentation through their transfer to adjacent keratinocytes. These structures can also grow hair shafts at the hair bulb following transportation from the Golgi region to the dendrite tips $(24,25)$. Melanocytes respond to environmental stimuli from surrounding keratinocytes to control differentiation and pigmentation, thereby determining hair and skin colors (26).

MITF is one of the key regulators of melanogenesis-limiting enzymes of the tyrosinase family, which include TYR, TYRP1, and TYRP2/DCT (27), The expression of MITF is modulated at the transcriptional level by specific transcription factors or at the post-transcriptional level by miRNAs (28). miR-143-5p can regulate MITF levels by targeting TAK1 (20). In the present study, it was demonstrated that MITF was upregulated in the presence of STTM-miR-143 in mouse melanocytes. MITF functions via a 'lineage addiction' mechanism to control specific aspects of the phenotypic expression of the melanocytic lineage and facilitate the transition of cells to a non-invasive stage. TYR and TYRP1 levels were decreased following miR-143 knockdown by STTM. However, DCT levels were increased following STTM-miR-143 overexpression. Therefore, it was hypothesized that MITF was not the only transcription factor involved in the regulation of TYRP2. In addition, the data were in agreement with those from a similar study demonstrating that TYR and TYRP1 levels were markedly decreased (27). The present study further demonstrated that TYRP2 expression was increased in MITF-knockdown melanocytes (MITF-KD) (27). Furthermore, TYRP2 expression levels were not regulated by the content of cellular melanin or by the levels of other regulatory enzymes in the melanogenic pathway (29). The mechanism of TYRP2 regulation has not been investigated to date. However, although MITF has been reported to be essential, it is not sufficient to induce the expression of melanogenic enzymes (30). In addition, it is a critical suppressor of innate immunity and can cause innate immune dysregulation of pigmentation, with implications in vitiligo, which is an autoimmune depigmentation disease (31).

During melanin transportation, melanosomes are transported across actin filaments via the association of the motor protein myosin Va with RAB27a and MLPH (32). RAB27a is a member of the Rab family of small GTPases, which are important regulators of membrane transport (33). Myosin Va requires simultaneous interaction with multiple components of a complex containing RAB27a and MLPH on the 

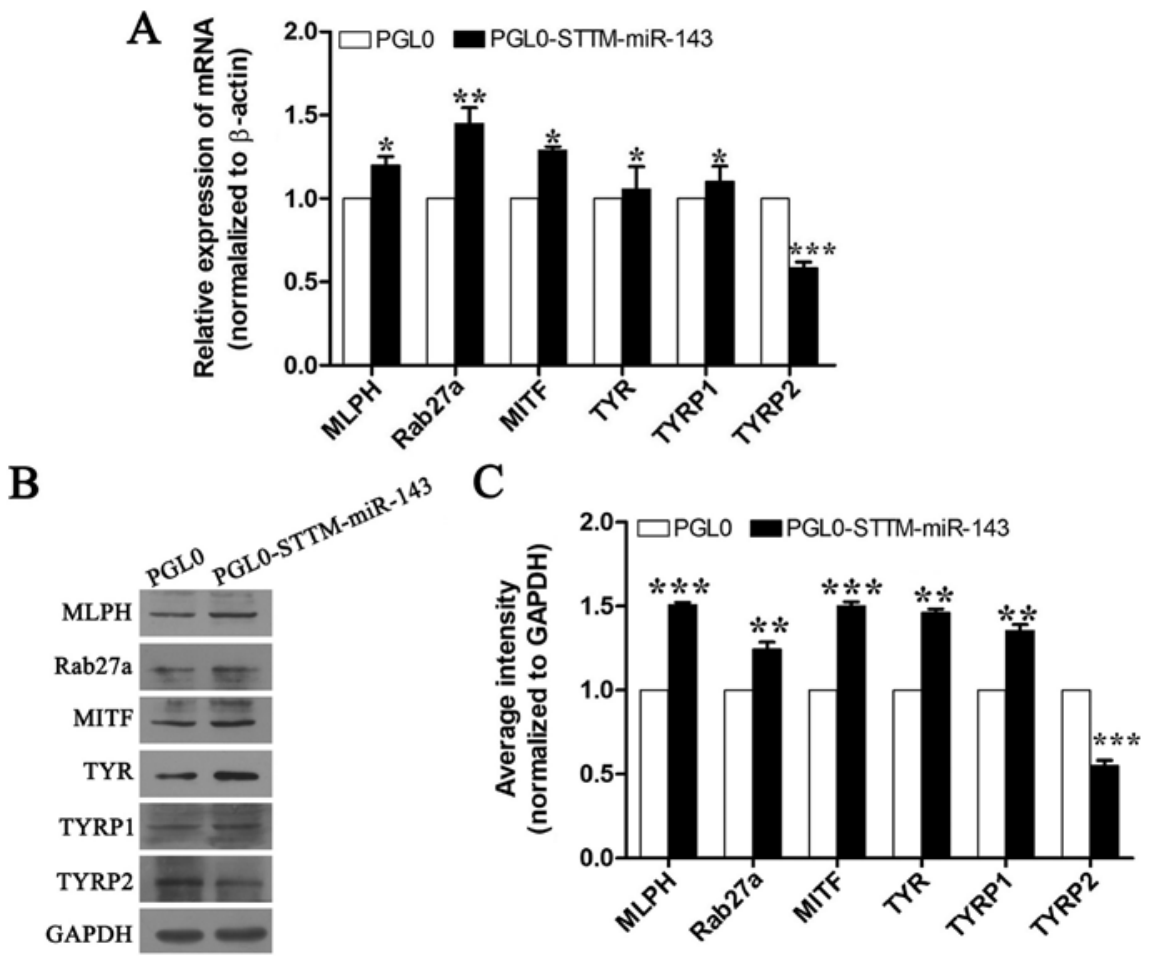

Figure 3. Effects of STTM-miR-143-5p on the expression of the melanogenic genes in melanocytes (A) mRNA expression of the genes $M L P H, R a b 27 a, M I T F$, TYR, TYRP1, and TYRP2 in mouse melanocytes transfected with the STTM-miR-143-5p expression plasmid. (B and C) Analysis of MLPH, Rab27a, MITF, TYR, TYRP1, and TYRP2 protein expression in melanocytes transfected with the STTM-miR-143-5p expression plasmids. The data were normalized to $\beta$-actin levels and expressed as the relative fold change. The data are expressed as the mean $\pm \mathrm{SD}(\mathrm{n}=3) .{ }^{*} \mathrm{P}<0.05 ;{ }^{* *} \mathrm{P}<0.01 ;{ }^{* * * *} \mathrm{P}<0.001$ vs. respective PGL0. STTM, short tandem target mimic; $M L P H$, melanophilin; MITF, microphthalmia-associated transcription factor; $T Y R$, tyrosinase; $T Y R P 1$, tyrosinase-related protein $1 ; T Y R P 2$, tyrosinase-related protein 2.
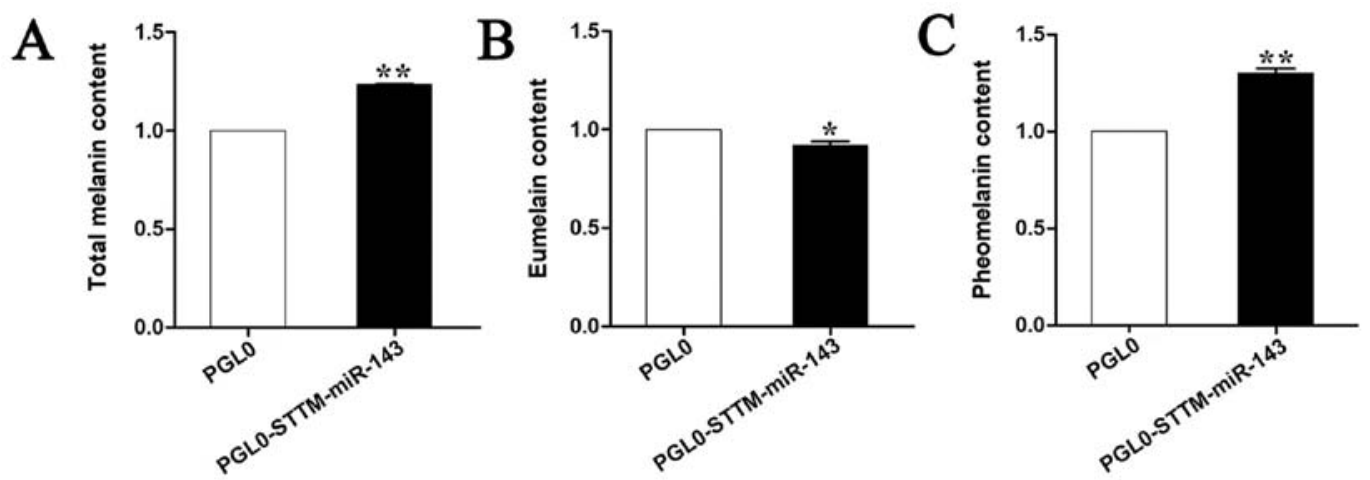

D
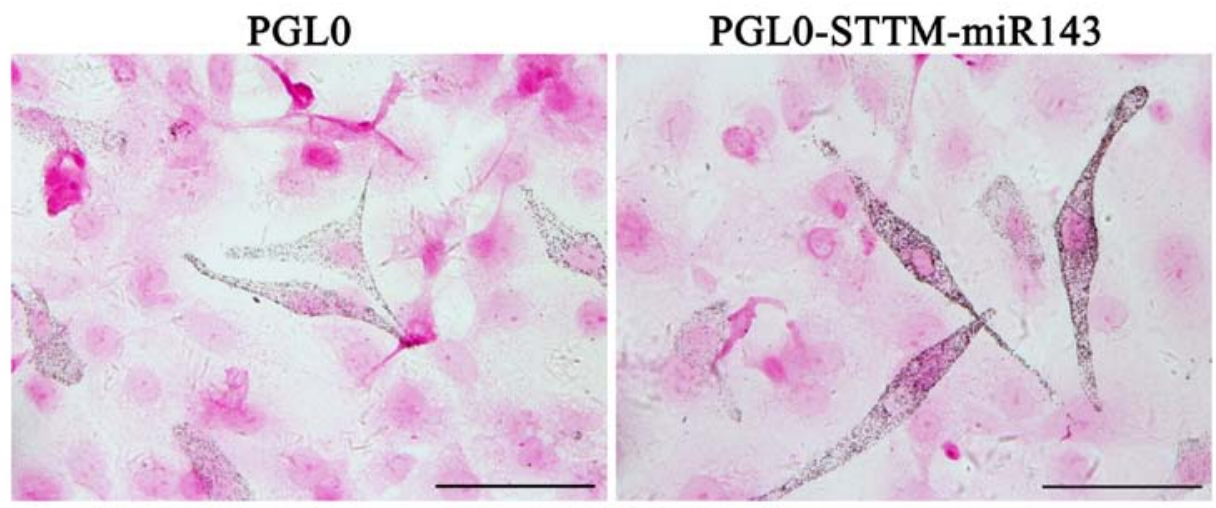

Figure 4. Effects of STTM-miR-143-5p on melanin production in melanocytes. Production of (A) ATM, (B) EM and (C) PM in mouse melanocytes overexpressing STTM-miR-143-5p expression plasmids. (D) Distribution of melanin in mouse melanocytes and keratinocytes by transfection of STTM-miR-143-5p or the negative control plasmids followed by Fontana-Masson staining. Scale bar, $200 \mu \mathrm{m}$. The data are expressed as the mean $\pm \mathrm{SD}(\mathrm{n}=3)$. " $\mathrm{P}<0.05 ;{ }^{* * *} \mathrm{P}<01 \mathrm{vs}$. PGL0. STTM, short tandem target mimic; ATM, alkali total melanin; EM, eumelanin; PM, pheomelanin. 
melanosomes (34). The C-terminus of MLPH can directly interact with actin, which is important for proper transportation and distribution of the melanosome to the dendrite tips (35). A positive correlation has been noted between the ability of MLPH to recruit MYO5A to melanosomes and the promotion of melanosomes in the peripheral retention (25). The accumulation of the pigment around the nucleus of the melanocytes and the abnormal transfer of melanin from melanocyte dendrites to keratinocytes can lead to albinism (36). miR-143 has been revealed to target MYO5A, and the present study demonstrated that STTM-miR-143 increased MYO5A levels and consequently MLPH and Rab27a levels, thus suggesting a stable interaction among MLPH, Rab27a and MYO5A. The absence of any one of these components may result in the failure of MYO5A to bind to melanosomes (34). In summary, STTM-miR-143 is an artificial biological tool used to control the melanogenesis by decreasing the expression of miR-143. This process regulates the levels of MYO5A, and in turn affects melanin secretion in melanocytes or in melanoma cells.

\section{Acknowledgements}

Not applicable.

\section{Funding}

The present study was supported by The Shanxi Scholarship Council of China (2017-072) and The Young Sanjin Scholars Distinguished Professor Program (RF).

\section{Availability of data and materials}

All data generated or analyzed during this study are included in this published article.

\section{Authors' contributions}

SQ and BL performed plasmid construction, cell culture and transfection, and gene expression experiments. JZ and XL performed melanin production, and analyzed and interpreted the data. CD and RF contributed to the conception and design of the experiments, and critically revised the manuscript for important intellectual content. SQ contributed to drafting the manuscript. All authors have read and approved the final manuscript and agree to be accountable for all aspects of the research in ensuring that the accuracy or integrity of any part of the work are appropriately investigated and resolved.

\section{Ethics approval and consent to participate}

Not applicable.

\section{Patient consent for publication}

Not applicable.

\section{Competing interests}

The authors declare that they have no competing interests.

\section{References}

1. Ito S and Wakamatsu K: Chemistry of mixed melanogenesis-pivotal roles of dopaquinone. Photochem Photobiol 84: 582-592, 2008

2. Vachtenheim J and Borovansky J: 'Transcription physiology' of pigment formation in melanocytes: Central role of MITF. Exp Dermatol 19: 617-627, 2010.

3. Levy C, Khaled M and Fisher DE: MITF: Master regulator of melanocyte development and melanoma oncogene. Trends Mol Med 12: 406-414, 2006.

4. Bertolotto C, Busca R, Abbe P, Bille K, Aberdam E, Ortonne JP and Ballotti R: Different cis-acting elements are involved in the regulation of TRP1 and TRP2 promoter activities by cyclic AMP: Pivotal role of M boxes (GTCATGTGCT) and of microphthalmia. Mol Cell Biol 18: 694-702, 1998.

5. Hume AN and Seabra MC: Melanosomes on the move: A model to understand organelle dynamics. Biochem Soc Trans 39: 1191-1196, 2011.

6. Matesic L, Yip R, Reuss AE, Swing DA, O'Sullivan TN, Fletcher CF, Copeland NG and Jenkins NA: Mutations in Mlph, encoding a member of the Rab effector family, cause the melanosome transport defects observed in leaden mice. Proc Natl Acad Sci USA 98: 10238-10243, 2001.

7. Kuroda TS, Ariga $\mathrm{H}$ and Fukuda M: The actin-binding domain of Slac2-a/melanophilin is required for melanosome distribution in melanocytes. Mol Cell Biol 23: 5245-5255, 2003.

8. Wu X, Sakamoto T, Zhang F, Sell JR and Hammer JA III: In vitro reconstitution of a transport complex containing Rab27a, melanophilin and myosin Va. FEBS Lett 580: 5863-5868, 2006.

9. Rudolf R, Bittins CM and Gerdes HH: The role of myosin V in exocytosis and synaptic plasticity. J Neurochem 116: 177-191, 2011.

10. Alves CP, Yokoyama S, Goedert L, Pontes CLS, Sousa JF, Fisher DE and Espreafico EM: MYO5A gene is a target of MITF in melanocytes. J Invest Dermatol 137: 985-989, 2017.

11. Au SY and Huang JD: A tissue-specific exon of myosin Va is responsible for selective cargo binding in melanocytes. Cell Motility Cytoskeleton 53: 89-102, 2002.

12. Tang G, Yan J, Gu Y, Qiao M, Fan R, Mao Y and Tang X: Construction of short tandem target mimic (STTM) to block the functions of plant and animal microRNAs. Methods 58: 118-125, 2012.

13. Bai R, Sen A, Yu Z, Yang G, Wang H, Fan R, Lv L, Lee KB, Smith GW and Dong C: Validation of methods for isolation and culture of alpaca melanocytes: A novel tool for in vitro studies of mechanisms controlling coat color. Asian Austral J Anim 23: 430-436, 2010

14. Chen C, Ridzon DA, Broomer AJ, Zhou Z, Lee DH, Nguyen JT, Barbisin M, Xu NL, Mahuvakar VR, Andersen MR, et al: Real-time quantification of microRNAs by stem-loop RT-PCR. Nucleic Acids Res 33: e179, 2005.

15. Livak KJ and Schmittgen TD: Analysis of relative gene expression data using real-time quantitative PCR and the 2(-Delta Delta C(T)) method. Methods 25: 402-408, 2001.

16. Dong C, Wang H, Xue L, Dong Y, Yang L, Fan R, Yu X, Tian X, Ma S and Smith GW: Coat color determination by miR-137 mediated down-regulation ofmicrophthalmia-associated transcription factor in a mouse model. RNA 18: 1679-1686, 2012.

17. Loftus SK: The next generation of melanocyte data: Genetic, epigenetic, and transcriptional resource datasets and analysis tools. Pigment Cell Melanoma Res 31: 442-447, 2018.

18. Cordes KR, Sheehy NT, White MP, Berry EC, Morton SU, Muth AN, Lee TH, Miano JM, Ivey KN and Srivastava D: miR-145 and miR-143 regulate smooth muscle cell fate and plasticity. Nature 460: 705-710, 2009.

19. Kim KH, Lee TR and Cho EG: SH3BP4, a novel pigmentation gene, is inversely regulated by miR-125b and MITF. Exp Mol Med 49: e367, 2017.

20. Ji K, Zhang P, Zhang J, Fan R, Liu Y, Yang S, Hu S, Liu X and Dong C: MicroRNA 143-5p regulates alpaca melanocyte migration, proliferation, and melanogenesis. Exp Dermatol 27: 166-171, 2018.

21. Liu B, Zhang J, Yang S, Ji K, Liu X, Du B, Jia Q, Qi S, Li X and Fan R: Effect of silencing microrna-508 by STTM on melanogenesis in alpaca (Vicugna pacos). Gene 678: 343-348, 2018.

22. Sommer L: Generation of melanocytes from neural crest cells. Pigment Cell Melanoma Res 24: 411-421, 2011.

23. Tian X, Jiang J, Fan R, Wang H, Meng X, He X, He J, Li H, Geng J, $\mathrm{Yu} \mathrm{X}$, et al: Identification and characterization of microRNAs in white and brown alpaca skin. BMC Genomics 13: 555, 2012. 
24. Jackson IJ: Molecular and developmental genetics of mouse coat color. Annu Rev Genet 28: 189-217, 1994.

25. Hume AN, Tarafder AK, Ramalho JS, Sviderskaya EV and Seabra MC: A coiled-coil domain of melanophilin is essential for Myosin Va recruitment and melanosome transport in melanocytes. Mol Biol Cell 17: 4720-4735, 2006.

26. Cichorek M, Wachulska M, Stasiewicz A and Tymińska A Skin melanocytes: Biology and development. Postepy Dermatol Alergol 30: 30-41, 2013.

27. Wang P, Li Y, Hong W, Zhen J, Ren J, Li Z and Xu A: The changes of microRNA expression profiles and tyrosinase related proteins in MITF knocked down melanocytes. Mol Biosyst 8: 2924-2931, 2012.

28. Hartman ML and Czyz M: MITF in melanoma: Mechanisms behind its expression and activity. Cell Mol Life Sci 72 1249-1260, 2015.

29. Pak BJ, Li Q, Kerbel RS and Ben-David Y: TYRP2-mediated resistance to cis-diamminedichloroplatinum (II) in human melanoma cells is independent of tyrosinase and TYRP1 expression and melanin content. Melanoma Res 10: 499-505, 2000.

30. Gaggioli C, Buscà R, Abbe P, Ortonne JP and Ballotti R: Microphthalmia-associated transcription factor (MITF) is required but is not sufficient to induce the expression of melanogenic genes. Pigment Cell Res 16: 374-382, 2003.

31. Harris ML, Fufa TD, Palmer JW, Joshi SS, Larson DM, Incao A, Gildea DE, Trivedi NS, Lee AN, Day CP, et al: A direct link between MITF, innate immunity, and hair graying. PLoS Biol 16: e2003648, 2018
32. Chang H, Choi H, Joo KM, Kim D and Lee TR: Manassantin B inhibits melanosome transport in melanocytes by disrupting the melanophilin-myosin Va interaction. Pigment Cell Melanoma Res 25: 765-772, 2012.

33. Zerial $\mathrm{M}$ and McBride $\mathrm{H}$ : Rab proteins as membrane organizers. Nat Rev Mol Cell Biol 2: 107-117, 2001.

34. Hume AN, Collinson LM, Hopkins CR, Strom M, Barral DC, Bossi G, Griffiths GM and Seabra MC: The leaden gene product is required with Rab27a to recruit myosin Va to melanosomes in melanocytes. Traffic 3: 193-202, 2002.

35. Kuroda TS, Itoh T and Fukuda M: Functional analysis of slac2-a/melanophilin as a linker protein between Rab27A and myosin Va in melanosome transport. Methods Enzymol 403: 419-431, 2005.

36. Marks MS and Seabra MC: The melanosome: Membrane dynamics in black and white. Nat Rev Mol Cell Biol 2: 738-748, 2001.

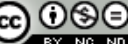

This work is licensed under a Creative Commons Attribution-NonCommercial-NoDerivatives 4.0 International (CC BY-NC-ND 4.0) License. 